Cisek, Drew \& Kalaska (Eds.)

Progress in Brain Research, Vol. 165

ISSN 0079-6123

Copyright (C) 2007 Elsevier B.V. All rights reserved

\title{
The place of 'codes' in nonlinear neurodynamics
}

\author{
Walter J. Freeman*
}

Abstract: A key problem in cognitive science is to explain the neural mechanisms of the rapid transposition between stimulus energy and abstract concept — between the specific and the generic - in both material and conceptual aspects, not between neural and psychic aspects. Three approaches by researchers to a solution in terms of neural codes are considered. Materialists seek rate and frequency codes in the interspike intervals of trains of action potentials induced by stimuli and carried by topologically organized axonal lines. Cognitivists refer to the symbol grounding problem and search for symbolic codes in firings of hierarchically organized feature-detector neurons of phonemes, lines, odorants, pressures, etc., that objectdetector neurons bind into representations of probabilities of stimulus occurrence. Dynamicists seek neural correlates of stimuli and associated behaviors in spatial patterns of oscillatory fields of dendritic activity that self-organize and evolve as trajectories through high-dimensional brain state space; the codes are landscapes of chaotic attractors. Unlike codes in DNA and the periodic table, these codes have neither alphabet nor syntax. They are epistemological metaphors required by experimentalists to measure neural activity and by engineers to model brain functions. Here I review the central neural mechanisms of olfaction as a paradigm for use of codes to explain how brains create cortical activities that mediate sensation, perception, comprehension, prediction, decision, and action or inaction.

Keywords: action-perception cycle; electroencephalogram; intentional arc; mesoscopic brain dynamics; neural code; phenomenology; reflex arc; scale-free cortical dynamics; wave packet

\section{Introduction}

Everyone knows the experience of smelling the scent of a rose. How does this happen? How do we interact with a material object and then know what it is and what it means for us? A neurobiologist says that we extract information from the chemicals and process it into a form suitable for comparison with information stored in memory; a cognitivist says that we make a representation and operate on the symbol according to certain rules; a

*Corresponding author. Tel.: + 510642 4220; Fax: + 510643 9290; E-mail: dfreeman@berkeley.edu, http://sulcus.berkeley.edu (W.J. Freeman). dynamicist says that we intend the rose. These words denote complex concepts that we use to describe an elementary process. We need to simplify. We know that we share the process with animals, which often have better acuity than we do, though not our depth of comprehension, so we can study the process in animals with brains less complex than our own. The same elementary process occurs in all our senses, not just the traditional five of sight, sound, touch, taste, and smell, but also gravity, muscle tension, muscle length, joint angle, and countless senses for chemicals concentrations, pressures, temperatures, and volumes throughout our bodies and brains. Olfaction is the most versatile and universal, rivaled only by the immune 
system, yet also the simplest and most ancient. It is the prototype for all other perceptual systems.

For these reasons olfaction in rabbits is a paradigm of choice for study to understand the ele-

mentary process (Freeman, 2001) in order to compare the biological, cognitive, dynamic, and philosophical descriptions of brain/mind function and find commonalities. We seek answers to the question: How can we so simply and elegantly cross the border between odorant and odor, between the material and the perceptual: in one direction to perceive the smell of a substance, in the other direction to create a chemical with a desired fragrance (Burr, 2002; Turin, 2006)? A concept of critical utility in this quest is "intentionality" (Freeman, 2007b); we must "intend" to perceive and create. By this we mean our minds using our bodies to thrust out into the world and in part change it and in part accommodate and assimilate to it by learning from the experience. The concept had its origin in the work of Thomas Aquinas describing the functions of mind and body; derivative meanings are the psychologists' "intent" meaning purpose and the analytic philosophers' "aboutness", the relation of a mental symbol to that which it represents (Searle, 1983). Aquinas further distinguished between "first intention", which is the perception of objects that need not be conscious, and "second intention", which includes awareness of the self-perceiving.

In this review an answer is sought in neurodynamics by analyzing patterns of neural activity that self-organize in the brain. This neural activity is hierarchically organized. Sensory inflows from receptors and motor outflows to muscles are by myriad pulses on axons at the microscopic level, the level of the "phantasms" of Thomas Aquinas and the inaccessible raw sense data of phenomenologists. These data require rate and frequency codes. Below is the flux of molecules at submicroscopic and quantum levels. Above is the self-organization of local fields into pulse and wave activity in spatiotemporal patterns at the mesoscopic level, the first and incomplete stage of perception where abstraction and generalization take place. Next is the organization of widespread fields of coordinated neural activity at the macroscopic level. The fields are large enough to include many areas of the brain, perhaps at times in synchronized oscillations involving the entire forebrain. At this level the perceptual contents in patterned activity include the locations in time and space of perceptions of objects and events. These patterns are not representations of stimuli, actions, thoughts, beliefs, etc.; they are expressions of knowledge in active support of perception, recollection, and decision. They do not result from computations in any literal mathematical sense. They are dynamic entities akin to vortices in hurricanes, unlike numbers in computers. We use symbolic codes to represent them and to model them with statistics and differential equations.

These levels are not intrinsic to brains; they are imposed by the scales required by the techniques used for measurements using chemistry, electrical recording, and brain imaging. The fallout for the synthesis by brain modelers is the necessity for bridging across these levels. For heuristic purposes I find that these three levels suffice: micro-mesomacro.

I postulate that these macroscopic self-organized goal states, through recursive self-similarity, include perceptions of present states, projections of future states, plans at the mesoscopic level for action to achieve them, and trajectories of microscopic pulses that direct muscular activity in goaldirected actions modulated by sensory feedback. This hierarchy gives the behaviorists' reflex arc, the pragmatists' action-perception cycle, and the phenomenologists' intentional arc. My proposed explanation in terms of central action through field neurodynamics is consistent with the nonrepresentational systems of Aquinas, Heidegger, and Merleau-Ponty that avoid the Cartesian subject-object split. I will conclude that, at present, neurodynamics can explain first intention - understanding perception as direct grasp of objects and events by animals and prelingual children - but lacks the experimental data on brain activity that will be needed to explain second intention whereby the self comprehends the immanent action of understanding itself. Field studies open a pathway to remedy this deficiency. 


\section{The neurodynamical paradigm}

Experimental neurobiologists are privileged in the search for understanding the process of transposition, because we have been granted the opportunity to record and measure the activity of neurons in the nose and in the many parts throughout the brain where the ongoing neural activity is modified by the elementary act that intends a rose, and represents it, and processes its information into knowledge. My group has recorded electrical activity from electrodes we fixed in the brains of rabbits trained to respond by sniffing or chewing after they learned the significance of simple odorant chemicals. By their actions we proved that they could identify the specific odorants that we presented to them. The rabbits acted the way they did because each time we presented an odorant we accompanied it by a reward or punishment that made the odorant meaningful for them. Without this reinforcement the odorants were meaningless for the rabbits, and they quickly learned to ignore them. With reinforcement they learned actions by which to get rewards and avoid punishments. They also learned to predict that any of several odorants would come in the near future, and they prepared their bodies to detect them and take appropriate action in response to whatever might occur, including the unexpected or unknown events, which in their limited and uncertain world could occur at any moment.

All these properties we derive from classical behaviorism. Psychologists describe and control these behaviors in terms of schedules of reinforcement (Ferster and Skinner, 1957); neurodynamicists describe them in terms of hierarchies of reflexes (Sherrington, 1906); philosophers describe them in terms of intentionality (Searle, 1983). Researchers comprehend the neural activity by recording and measuring the electric potential differences in and around the brains of the animals (e.g., scalp EEG, the magnetoencephalogram, MEG) as they anticipate, detect, and respond appropriately to the odorants in their learned repertoires. There is a notable reciprocity between the intention of a rabbit to perceive a signal of import and the intention of a researcher to perceive the neural activity. The animal prepares its body by orienting its nasal sensory receptors and sniffing; the researcher prepares and places electrode arrays, rigs electronics to amplify, filter and measure signals, and creates displays to bring the measurements to the observer's senses. The designs of the arrays, the filters, and the methods for measurements to extract information depend on the expectations of the researcher and the properties of the subjects. The details are complex and of interest only to specialists, but in principle the process is the same in man and rabbit. From our respective experiences we and our rabbit predict what the future holds; we plan appropriate tests of our predictions; we make the tests and detect the changes in our sensory input that are caused by our actions in making the test; we classify the results of our test by whether or not what happens conforms with what we expect to happen; and we modify our expectations accordingly. We and they are not observers; we are participants in a circumscribed relationship.

Of course, the rabbit is much simpler, and therein lies its utility. From its training it expects to receive any one of two or three odorants at some time in the near future, and it samples the air each time it breathes in. When an odorant comes, the rabbit detects it with its nose, determines with its brain which expected event has occurred, and with its body takes appropriate action such as sniffing or chewing or relaxing. The crux of the problem lies in the neural events by which the determination occurs in the brain of the odor from the odorant. We divide the neurobiological process into stages. In the first stage we observe the effect of the odorant on the receptor cells in the nose. In the second stage we observe the effect of the activated receptors on the olfactory brain. In the third stage we observe the effect of the olfactory system on the whole brain. Fourth, we observe the neural activities in the motor systems. Lastly we observe the effect of the brain on the body, as the rabbit responds to the odorant. The transposition from odorant to odor occurs in the second and third stages. We observe the process in these stages with electrodes in the brain by which to record, measure, and model neural activity, first in the olfactory system, then in the neocortices serving the 
1 limbic system and the other distance receptors in the eye, ear, and skin.

\section{The network approach: information processing and linear causality}

Consider again that we are contrasting the reflex arc with the intentional arc. The intentional arc begins with emergence from the present brain state of an extrapolation into the future that will require some appropriate action to direct the self into successful assimilation with an altering world. That foresight includes prior specification of what information might be needed through acts of observation and perception to achieve success. The details are formulated in the attractor landscapes emerging through preafference. The reflex arc is widely thought to begin with the stimulus that activates the sensory systems of a subject and to end with the response. To the contrary the reflex arc begins with the intentional action of the observer to explore the properties of the subject. The "features" of the stimulus emerge in the mind of the experimenter and are embodied in the selection and delivery of the stimulus. Neural correlates of the "features" are clearly detectable in the evoked activity of the brain, but whether and how the brains of subjects transform these evoked patterns of activity into percepts are matters for investigation. The aim of electrophysiological investigation is proposed here as challenging the "feature detector" concept and offering the "attractor landscape" concept as an alternative.

Each electrode inserted into the nose or the brain yields two forms of electrical activity. We see one form in trains of electric pulses (spikes, action potentials, units) from individual neurons. We see the other form in continuous waves of electric current (dendritic potentials, local field potentials, electrocorticograms - ECoG, scalp electroencephalograms - EEG) from populations of neurons. The study of pulses is based on the view of the organization of olfactory receptors and brain areas as networks of spiking neurons. The study of waves is based on the view of the same neurons generating continuous space-time fields, in which the identities of the neurons are submerged in the populations. The differences in views resemble those between the psychological analyses of individuals in families contrasted with sociological analyses of the organizations of cities and nations. At the start of the neurobiological experiments the electrodes are shaped and placed to maximize the detection of either pulses or waves, and the recordings of electrical activity containing both forms are filtered to separate the pulses and the waves for analyses. The data from each stream are used to construct hypotheses about the functions of the olfactory brain, on one hand as discrete networks of neurons that are connected by junctions, the synapses, and on the other hand as tissues that contain such high densities of neurons and synapses that the tissue can be described as a continuum, analogous to ways in which molecules can be described as forming a liquid or gas, and supporting both synaptic and nonsynaptic communication and modulation (Freeman, 2005c).

A selective synthesis of both views is essential for understanding brain function. This is because brains work at many levels of organization. An act of perception involves all levels of activity, ranging from the attachment of individual molecules of an odorant to the molecular structures on the surfaces of olfactory receptor cells to the initiation by the rabbit of sequences of social behaviors intended to enhance the likelihood of its species to survive. The guiding principles of experimental neurobiology are that we record activities of both kinds as the neural correlates of the process by which an odorant is comprehended as an odor, and that we use our observations of the correlates to construct explanations in the form of dynamic models of the brain systems that perform the process. Notably these numerical correlates interrelate patterns of neural activity with patterns of goal-directed behavior, not with consciousness or verbal descriptions of phenomenological states. We have no measure of what rabbits feel or what they are conscious of. We deal here with the process of inductive category formation in the accumulation and intentional utilization of knowledge, for which emotion is an integral part (Freeman, 2001), not with the 'hard problem' at the core of consciousness studies (Chalmers, 1996). 
The network model is commonly assumed to begin with the reflex arc (but see "The continuity of circular causality across all levels"), in which the stimulus has the form of molecules of odorant that

bind to receptor cells at the molecular and quantum levels. The binding releases a wave of electric current, the generator potential, that initiates and sustains firing of pulse trains from just those receptor neurons that can selectively bind the molecules. According to various authors (Lettvin and Gesteland, 1965; Lancet and Ben-Arie, 1993; Freeman, 2001; Burr, 2002; Buck and Axel, 2004) the microscopic neurons encode sensory information in their pulses and transmit it by axons into the olfactory brain, where it is directed by switching networks to selected neurons that by filtering or resonance act as feature detectors. The cortical neurons send the processed information to associational areas of the brain.

The steps beyond are conjectured from properties of artificial neural networks: higher areas are thought to compare the input information with previously stored information retrieved from memory by symbolic dynamics. Studies of perception in humans report the firing of neurons with remarkable specificity to stimuli such as photographs of famous persons (e.g., Quiroga et al., 2005), suggesting that their spike trains serve as symbols. Cognitivists propose that the best matching symbol is selected by competitive inhibition among such neurons and sent to the motor cortex, where an appropriate response is selected by winner-take-all for transmission into the motor systems of the brain stem and spinal cord. All this must occur in time frames lasting on the order of half a second.

\section{The field approach: the action-perception cycle}

The field theoretic model using the action-perception cycle begins not with the stimulus but instead with the formation in the forebrain of a macroscopic pattern that embodies anticipation of a desired future state of the brain and body, such as finding food or avoiding danger. We conjecture that within this macroscopic pattern the brain constructs mesoscopic activity patterns, which organize the local sensory and motor populations that control the actions intended to achieve the goal. Within each mesoscopic population the microscopic neurons are directed ("ordered") to fire pulses in prescribed sequences. These individual neurons also receive proprioceptive feedback from sensory receptors in the muscles and joints through the cerebellum and basal ganglia that is needed to continuously adapt the intended movement of the body to the intended goal. Knowledge about the neurobiology of these two downward steps is insufficient to detect and measure the mesoscopic patterns. They can be conceived in engineering terms as predictive systems such as those for controlling the flight of an airplane, which have an over-arching level in which the goal is selected by choosing a flight plan, outer loops that set the control surfaces to direct the aircraft to its goal, and inner loops that regulate the control surfaces in the wings and tail to compensate for air turbulence. In these terms the macroscopic pattern establishes a context embedding the mesoscopic patterns that self-organize in multiple populations comprising 'modules' (Houk and Wise, 1995; Houk, 2005), and the modules establish the local contexts in which microscopic neural networks perform the intended tasks.

The movements of the body in every intended overt action modify the positions with respect to the environment of the receptor cells in all sensory systems. The modifications change the sensory input. These self-induced changes are anticipated and predicted from past experience. The predictions have been described as communicated from the motor modules to the sensory modules of the brain by copies of the motor outflow known as "corollary discharges" (Sperry, 1950) and "efference copies" (Von Holst and Mittelstädt, 1950) in the process of "preafference" (Kay and Freeman, 1998), which is the basis for focused attention. The corollary discharges prime the sensory areas by making them selectively sensitive to each of the expected stimuli in the search for odorants signifying food or danger, be they from carrot or fox, cabbage, or man.

Studies of neural fields (Freeman, 2004a, b, 2005a, 2006a) show that the impact of the pulses from the receptors on the sensory areas of the 
1 brain is not at all the processing of spikes on a few hundred axons. In olfaction the millions of pulses

with each inhalation cause a major change in function, which is equivalent to the change in state from a gas to a liquid (Freeman and Vitiello, 2006). The nearly random activity before the impact is increased in amplitude, and at some point it condenses much as would water molecules forming a raindrop. In physical terms the impact induces a phase transition in the olfactory brain, which forces it out of its receiving state that is maintained at a pseudoequilibrium (Freeman, 2005b) into a transmitting state into which the bulbar dynamics converges. The transition period leading to convergence is a brief metastable state (Bressler and Kelso, 2001) of search through the selective classes of sensitivities stored by modifications of synaptic strengths from prior learning in an attractor landscape. We conceive each cortical dynamical system as having a state space through which the system travels as a point moving along a path (trajectory) through the state space (Kozma and Freeman, 2003).

A simple analogy is a spaceship flying over a landscape with valleys resembling the craters on the moon. An expected stimulus contained in the omnipresent background input selects a crater into which the ship descends. The convergent region in each crater defines the attractor to which the system trajectory goes, and the set of craters are the basins of attraction in the attractor landscape. There is a different attractor for each class of stimulus that has been learned and that preafference has primed the system to anticipate, each surrounded by its basin. The landscape is surrounded by a catch basin that signals unknown stimuli (Skarda and Freeman, 1987) that might be important. These output patterns trigger a fixed "auto-shaped" behavioral action known as the "orienting response". The animal receiving an unexpected stimulus freezes and directs its senses in search of something unknown and possibly threatening. If the unknown stimulus is accompanied by reinforcement, then a new attractor forms by Hebbian learning, which changes all of the other basins in deforming the landscape by attractor crowding. If there is no reinforcement, the system automatically adapts by habituation to block cortical responsiveness to that input in the future. These processes of Hebbian linkage and non-Hebbian habituation are the essence of associative memory. There is an exclusion principle at work in that only one attractor can be selected at a time (Freeman and Vitiello, 2006), though rapid rotation among two or more attractors may occur. Sequences of patterns indicate that "itinerant trajectories" (Tsuda, 2001) form through successions of attractors in the landscape, each attractor dissolving as soon as it is accessed and giving way to the next.

The dynamics in each sensory cortex (not just for olfaction but also vision, hearing, and touch) converges within milliseconds to an attractor, which transmits a modality-specific burst of neural activity that I call a "wave packet" (Freeman, $1975 / 2004,2000)$ This is a spatially coherent oscillation of dendritic potentials typically in the gamma range $(30-80 \mathrm{~Hz})$ with relatively fixed spatial patterns of amplitude and phase modulation (AM, PM) of the shared wave form (Freeman, 2004b, 2007a). The perceptual contents of the AM patterns are determined by the previously learned synaptic connections in the sensory cortices, which constitute the integrated record of knowledge constructed during prior experience with the stimulus. That synaptic network determines the attractor and its basin in the landscape sustained by each cortex for each learned class of stimulus. A Hebbian network spans the basin of each class. The stimulus-evoked action potentials that are triggered by an expected stimulus select a basin by activating the network; this is the process of generalization to the class of the detected stimulus as the trajectory converges to the attractor, irrespective of where the cortex was placed within the basin by the particular receptors that the stimulus attached to, which vary from trial to trial. With each trial the process of learning continues to refine and update the Hebbian synaptic network. As the system converges to the attractor in the basin, it deletes the extraneous information about which particular receptors receive the stimulus; this is the process of abstraction. The attractor determines the transmitted wave packet, not the stimulus, which merely selects and refines the transmitted AM pattern, which is an expression of its 
knowledge by the rabbit that in terms of coding can be modeled as a symbol of its contents.

Owing to the large surface area of sensory cortex that is integrated by the attractor (Freeman, 2004b) and the divergent-convergent topology of the transmitting bundles of axons, the patterns are broadcast through the brain. Those cortical transmission pathways that have divergent-convergent projections and not topographic mapping perform a spatial integral transformation on the output. Transmitted activity having dispersed phase and frequency values is attenuated by cancellation and smoothing; activity that is spatially coherent (same frequency and phase) is relatively enhanced. The most salient among the targets of transmission is the limbic system. This is the core structure of every vertebrate brain that is identified with the expression of emotion. Its key structure, the hippocampus, was the first cortex to appear as laminated neuropil in the phylogenetic evolution of the brain (Maclean, 1969), and it well deserves its appellation, archicortex ("ancient cortex"). The hippocampus sustains the neural machinery by which sensory events and objects are assigned environmental spatial locations and times of occurrence in the stream of life history (Freeman, 2001). In mammalian brains the wave packets of all sensory cortices are received either directly from the olfactory bulb or by relays from other modalities by the hippocampal vestibule, the outer layer of the entorhinal cortex. Time and place are linked to each other and to the contents of multimodal stimuli (Gestalts) in the hippocampus. There the multiple sensory cortical wave packets are integrated into a multisensory pattern as they pass through the hippocampus back to the deep layers of the entorhinal cortex, whence it is disseminated back to the cortices of origin. Every event must make this passage, if it is to be assigned a spacetime location in the stream of personal history.

These properties are commonly referred to as the spatial "cognitive map" and the temporal "short term memory" provided by the hippocampus (O'Keefe and Nadel, 1978; Buzsaki, 2002). The collective and incremental modification is the basis for self-assimilation by which the animal continuously updates its tenancy in the environment. The combined spatiotemporal pattern that

is assembled in the hippocampus is re-transmitted by stages to all sensory areas by preafference. The result is that within half a second of the original event there emerges in the brain a global pattern of cortical activity that is participated in by every sensory area (Freeman and Burke, 2003: Freeman and Rogers, 2003). I postulate that this global pattern updates the contents of attractor landscapes, implement the prediction of new sensory inputs, and issue fresh motor commands. Preafference operates not as copies of motor commands for error correction (Von Holst and Mittelstädt, 1950) but by participation in a macroscopic, spatially coherent AM pattern. This emergence of the macroscopic pattern completes the action-perception cycle with assimilation (Freeman, 1995), literally within the time frame required for the blink of an eye.

\section{Circular causality}

One may ask where in the brain does one see the macroscopic pattern and in what form? My answer is that it appears in synchronized oscillations over broad ranges of beta frequencies in ECoG (Freeman and Burke, 2003; Freeman and Rogers, 2003) and EEG (Freeman et al., 2003a, b) and ECoG (Freeman and Burke, 2003), and that the underlying activity organizes all parts of brain and body that are simultaneously engaged with the material, formal and social environments. To focus again on olfaction, the molecular structures of the receptor cells in the nose are active in binding odorant molecules from the air stream. So also are the myriad synapses in the sensory and motor areas of cortex and at the neuromuscular synapses on muscle cells, which bind neurotransmitter molecules at the submicroscopic level. The networks of neurons in the olfactory brain are active in preprocessing the information delivered by pulses from receptor cells into cortical networks, executing the essentially engineering operations of amplification, range compression, normalization, filtering, and selective enhancement of the information (Freeman, 1999). The entire olfactory brain is reorganized in a phase transition by which the stimulus selects the class to which it belongs, and the entire 
1 olfactory system transmits a wave packet throughout the basal forebrain including the limbic system. The subsequent formation of a macroscopic pattern integrates the activity of the entire forebrain including the limbic, motor, and olfactory systems (Freeman and Burke, 2003). I conjecture that the pattern provides the context in which the appropriate behavior self-organizes, containing the trajectories of microscopic neural activity and mesoscopic limb movements that are required to achieve emergent goal states. Molecular, cellular, and mesoscopic assemblies are modulated and directed at all times, everywhere, and at all levels. How might this orchestration take place?

One might ask a similar question about any large-scale, self-organized physical process such as a hurricane or a tree. How does each molecule of air or water conform its trajectory into the gigantic vortex that feeds on solar energy? How does each pore on every leaf in the sunlight coordinate with every hair on every root branch in the ground? Correspondingly, how does each molecule of neurotransmitter substance and each neuron and each local assembly conform to the global organization that we observe in animal and human behavior? These questions we can answer now by combining neurobiological observation and experimentation with theory from physics, chemistry, and mathematics (Prigogine, 1980; Haken, 1983). But hurricanes and trees cannot intend, whereas brains can and do intend. The difference is twofold: hurricanes and trees cannot remember and utilize their past, and neither trees nor hurricanes can direct the movement of their bodies through their environments. They have no brains. Only animals with brains have the machinery for anticipating future states, planning for deployment of their bodies in pursuit of satisfaction of perceived needs, predicting the consequences on sensory inflow of their own actions, and above all for selfassimilating by which they bring their brains and bodies into conformance with their environments. In short, hurricanes and trees lack the mechanisms required for intentionality (Freeman, 1995).

It is immediately apparent that intention spans the entire range of material, psychological, and social behaviors, from the most distant conception of survival and procreation to the molecular changes in nerve and receptor cells that enable sensation, learning, and muscle contraction. The material basis at each level and its teleological relations to levels immediately below and above are well described by the particular science that is directed to the level. Of particular concern is the relation between levels that is described with the concept of circular causality (Haken, 1983): in selforganization the higher level order forms by the interactions of lower order parts. The now classic example in physics and engineering comes from the dynamics of a laser. The parts are the atoms in a gas that oscillate at frequencies in a wide distribution about some mean value, when they are in a state of low energy. When energy is pumped into the atoms, they oscillate more strongly and interact with each other more strongly. At some threshold they undergo a state transition and oscillate all at a shared frequency. The high-energy oscillation is called an "order parameter", because the atoms that generate the oscillation are "ordered" ("enslaved" according to Haken) by the whole to oscillate at one frequency. The reason this process is described as "circular causality" is that the particles (neurons) create the field (the wave packet) and the field imposes order onto the particles. Similarly in the olfactory brain at low energy before a stimulus input arrives, the neurons emit pulses seemingly at random with a distribution of pulse frequencies. When their energy level is increased by excitation from olfactory receptors, their pulse frequencies increase. At some threshold the whole population interacts so strongly that all the neuronal potentials oscillate at the same instantaneous frequency, though with different amplitudes and different levels of participation. The population signal seen in the AM pattern of the wave packet in that frequency range is an order parameter that brings all of the neurons into varying degrees of synchronous oscillation (Freeman and Vitiello, 2006).

The analogy is limited, because atoms are all indistinguishable, whereas neurons all differ from one another, no two being identical. Whereas all the atoms are locked into the one order parameter, the neurons in a population have varying degrees of sharing in the common signal; the order parameter is vectorial. Owing to their individual 
differences the classical descriptions from statistical mechanics are not adequate to describe population neurodynamics. Descriptions using concepts from classical thermodynamics certainly

apply in terms of the requirements for disposal by brains of waste heat and entropy, as well as essential constraints on brain temperature, pressure, mass, and volume that are self-regulated. The analogy does have great value, because it expresses a fundamental property of brains in a simple way: populations of neurons interact by recurrent excitation and inhibition through synaptic transmission and create order parameters that regulate the same neurons. This is "circular causality". It differs from "entrainment", which denotes the reciprocal interaction of two entities at the same level, such as clocks or neurons. As introduced by Haken (1983) it denotes the conformance of the individual with the group, which requires field effects as seen in mobs and vortices. We observe the individual neural activity in pulse trains on axons; we observe the order parameter in waves of dendritic currents. The relation between pulses and waves is bidirectional. We predict the wave densities from pulse densities by averaging over the parts that form the whole. We deduce the effects of the waves on the pulse densities by calculating differences in wave densities. Integration carries us to the higher level; differentiation carries us to the lower level. These processes of summation and differencing occur simultaneously in all areas of cortex (Freeman, in press). The predominant direction of information flow through these processes in sensory areas is upward from individual neural activity to population densities; the predominant direction in motor areas is downward from population wave densities to more individually structured trajectories of pulse densities.

\section{The continuity of circular causality across all levels}

Looking downwardly, neurons are microscopic parts of mesoscopic populations, yet each neuron is a semi-autonomous whole that develops and maintains complex relations among its parts. It devotes most of its lifespan to its own janitorial functions; the typical cortical neuron fires a pulse lasting $1 \mathrm{~ms}$ at an average rate of $1 / \mathrm{s}$, which would scale to 1 full day every 3 years. Yet it is ceaselessly active at all times in responding to input from an average 10,000 other neurons (Braitenberg and Schüz, 1998) by which it is modulated through multiple order parameters. Each of its parts is a subwhole, which is organized by assemblies of macromolecules that provide the energy for generating electric fields, opening and closing ion channels, and maintaining chemical balances. Each macromolecule is an organized assembly of atoms that performs a designated task that depends on collective, patterned action expressing an order parameter. Looking upwardly, mesoscopic neural populations are components of ongoing macroscopic fields comprising organized actions of the whole brain. The brain is one organ among many in the body that cooperate continually in directed actions. The body is embedded in organic relations with the material and social worlds, and so on. Each of these levels generates order parameters at differing scales of time and space, and operates with entities, states, and state variables that are unique to the point of view taken by scientists engaged in systematic study at each level. Yet brain wave dynamics is scale-free (Freeman, 2005b, 2007c), meaning that its wave patterns of electrical activity are self-similar (Barabásí, 2002) across wide scales of time and space, as shown by measurements of distributions of its dynamic properties, most obviously those of the neocortex (Freeman, 2006b). It is the scale-free dynamics that appears to enable mammalian brains varying in mass $10^{4}$ from mouse to whale to participate in and organize all levels of function simultaneously by transactions that extend seamlessly across the entire range, yet which can be abstracted for measurement and analysis at each desired level with its pertinent scales of measurement. These measurements give the numbers that are translated into information, and the numbers support the analyses by modeling based in symbolic codes.

The reflex arc actually begins not with a stimulus but with the intention of the investigator, who selects and delivers a stimulus to the subject with the goal of constructing a useful code. The stimulus is a pattern of chemical energy that impacts on individual receptors at the atomic level with 
1 binding of molecules of scent to the surfaces of receptor cells, initiating cascades of biochemical reactions resulting in microscopic pulses transmitted to the brain. The impact of myriads of pulses with inhalation destabilizes the olfactory brain and changes the order parameter to an intracortical search mode. Convergence to an attractor means that the collective population of neurons enters into an ordered state that modulates the pulse trains of the entire olfactory brain, sending an AM microscopic pulses to other parts of the brain. The pattern of the wave packet, being mesoscopic, is not detectable by observing the pulse trains of any small number of neurons; it is only seen in large averages. The integration of multiple wave packets supports the emergence of a global brain state that provides an order parameter that includes the motor areas simultaneously with the sensory areas. This macroscopic context modulates the mesoscopic populations that organize the motor areas into controlled sequences of oscillations and shape the sensitivities of the sensory areas by selection of attractor landscapes in preafference. The reflex arc is completed by the modular organization of microscopic pulse trains of motor neurons, which release the neurochemical synaptic transmitter molecules that are required for muscle contraction.

Whatever the intent of the investigator, the intentional arc of the animal begins with its intention as expressed in its macroscopic goal state and extends through mesoscopic patterns to the microscopic level of muscle contraction. Actions cause changes in the microscopic binding of chemicals to chemoreceptors, photons to visual receptors, and so on, which are transduced into rates and frequencies of firing, followed by mesoscopic phase transitions and, eventually by closure of the arc on assimilation and updating of the perceptual wave packets and the conceptual macroscopic state. This is the action-perception cycle. pattern that is carried by the patterns of myriad
From sensation to perception to conception; from goal to plan to action

The above descriptions of the neural correlates of intentional action and perception, when viewed in terms of scale-free brain dynamics across the broad range of scientific disciplines, leads to the view that engagement of the individual with the environment is simultaneous at all levels. Even though there are no "atomic propositions" (Barlow, 1972), the metaphors for coding are invaluable for communication among researchers. The material engagement takes place in the immersion of body and receptors in gases, liquids, and solids governed at the atomic and molecular levels by quantum field theory, and at macroscopic levels by Newtonian physics through forces that modulate the firings of stretch receptors in muscles, pressure receptors in skin, joints internal organs, and vestibular receptors for gravity and acceleration of the head. These chemical and physical forces permeate brains and bodies with continuous presentation of information to the brain, followed by its selective distillation into knowledge. At the mesoscopic level there is preconscious apprehension of the influx of new relationships between body and environment that go far beyond information processing in the emergence of wave packets, which can be interpreted as symbols of generalizations representing confirmations or disclaimers of anticipations regarding the continuity of the fabric of the world and the place claimed by the individual, the "horizons" of Merleau-Ponty (1942/1963). These surmises about the impending future accompany the preparations for rest or for incipient action to deal with predicted or unexpected contingencies in the surround, the arena of perception. Yet this is not all. Embedding the perceptual and premotor activities of body and brain is the guiding matrix of goals, ranging in scope and complexity from what to do in the next few seconds in the face of opportunity or danger to lifelong ambition to flourish and prevail. It is this selfstructured dynamic edifice of anticipations rooted in the accumulated self-assimilations of a lifetime of knowledge that modulates, enriches, and integrates the experience so immediately reflected in mesoscopic and macroscopic patterns of brain 
1 activity. We have also discovered their traces in electrical fields at the surface of the human scalp (Freeman et al., 2003), but we cannot yet read them, because we do not yet know how to encode their patterns in terms of information and symbols adequately to correlate them with behavioral measurements that include verbal communications.

This description of intentional brain dynamics was pioneered seven centuries ago by Aquinas

11 (1272), who dismissed the passivity of the Platonic soul by conceiving intention as taking action (in-

13 tendere) and coming to know the world by selfassimilation (adequatio), which is conforming the

15 body and brain with the environment and not the Aristotelian processing and storing of forms (in-

17 formation). In the view of the intentional arc the goal pre-exists the action, whereas in the view of the reflex arc the goal exists as an achievement after completion of the action. According to Aquinas (Q 85, A 2) there are two kinds of intentional action. One is transitive action in mechanistically thrusting the body into the world in the manner of a robot or other machine. The other is immanent action by understanding, which distinguishes the actions of animals and humans from those of machines that act without comprehending what they are doing. Understanding includes contemplative withholding of action but still has reference to or engagement in the world that provides knowledge through self-assimilation through learning from the senses, herein differing from idealist conceptions that understanding is derived solely through reference to innate codes in the brain. Understanding does not occur at the microscopic level of single neural activity of pulses, which is unique and ephemeral and directly related to the particular stimulus that drives it. These Aquinian phantasms are likenesses of a thing and not the thing, in the manner that trains of action potentials that bear information to the brain are the likeness of a stimulus but not the stimulus. Being unique events, the phantasms (the patterns of the microscopic pulses, the raw sense data) are unknowable.

The mesoscopic level is that of the intelligible species, which forms by abstraction and generalization over multiple sequential phantasms. Here is the first step of crossing from the realm of the material to the realm of the perceptual, from the concrete to the abstract. The transposition begins in sensory areas with modality-specific wave packets, which embody a selection of all stored experience that is immediately relevant to the intended inputs (The information-bearing stimuli that are sought by intentional observations). The wave packets are not fully intelligible, because they lack multisensory integration and orientation in time and space from convergence and passage through the limbic system. Aquinas wrote (Q 79, A 4): "Therefore we must say that in the soul is some power derived from a higher intellect, whereby it is able to light up the phantasmata. And we know this by experience, since we perceive that we abstract universal forms from their particular conditions, which is to make them actually intelligible." His "light up" appears to correspond to the stage of self-assimilation when a macroscopic state emerges following the limbic integration of mesoscopic wave packets and preafference (Freeman and Burke, 2003; Freeman and Rogers, 2003). That macroscopic order parameter modulates all sensory cortices and includes the motor areas, which must be engaged in the process of deciding what to do in the light of new integrated input stemming from the senses.

The new state of knowledge is an engagement with the situation of brain and body in the world that by self-similarity contains mesoscopic preparatory states in both sensory and motor areas for planning action and predicting its sensory consequences. By virtue of scale-free dynamics the engagement occurs at all levels simultaneously, they may be material, formal, or social. Through mesoscopic and macroscopic constructions the brain conceives, grasps, and approaches by sequential actions with the body what Merleau-Ponty called "maximum grip" immediately and directly in the way that an aircraft pilot, a car driver, and a tennis player experience the instruments as extensions of the body, not as inner manipulation of symbols and representations or exercise of codes in computational logic. This elemental process does not posit consciousness; there is no need at this level for that hypothesis. Self-awareness in these actions is by neural mechanisms not yet adequately 
examined in humans to provide the experimental field data required to build the appropriate theory, but it readily appears that the recursive embedding provided by circular causality in macroscopic patterns of transient global synchrony will be identified as crucial in the process of consciousness.

\section{First intention and second intention}

This description of the neurodynamics of intentionality has been made possible only in the past few years, equally by advances in technology that enabled simultaneous EEG recording from large electrode arrays implanted onto the surface of the brain or on the scalp of humans, and by advances in theory that enabled modeling the EEG patterns using concepts from nonlinear dynamical theory (Freeman and Vitiello, 2006), neuropercolation theory (Kozma et al., 2005), and scale-free dynamics (Barabásí, 2002; Freeman, 2006a, 2007c). These developments open the way to reconsider long-standing differences between cognitivists and phenomenologists in their interpretations of intentionality. Descartes abandoned the Thomist concept of intentionality in his dualist, subject-object description of the soul operating the brain like a pilot controlling machine functions using representational logic and mathematics. Intention was re-introduced by Brentano (1889/1969) as the basis for distinguishing the representations and operations on them of humans who know what they are doing from those of machines that do not know. The usages by his successors have led to Searle's (1983) characterization of intentionality as "aboutness", because a thought or a perception is "about" something. This interpretation suffers the intractable difficulty of grounding coding symbols in machines and brains to the entities they represent. For example, what is the relation between a word in a computer memory and the real person it represents? Similarly, how does the firing of neurons in the cortex of the fusiform gyrus signify the perception of a face, and how does that firing "cause" one to classify the person whose face it is?

Heidegger (1975/1988) reintroduced what he called "the enigmatic phenomenon of intentionality" in a form that is indistinguishable from that of Aquinas, despite his denial of any indebtedness to the "Scholastics". The only reason for citing his turgid, obfuscatory, quasimystical work for neuroscientists is that he addressed what he rightly called "the central problem of philosophy", the same as that with which this review began: in his terms, “... the 'transposition' [transcendence] of the Dasein over to things", and that he led other phenomenologists, principally Merleau-Ponty, back to this forgotten insight. By "Dasein" he simply meant the underlying, largely unconscious, intentional self and not the egoistic awareness of self. He usefully distinguished two widespread "misinterpretations" of "intentionality". First was the "common sense" assignment of intentionality to the subject; Searle (1983) wrote that the firing of neurons caused perception of an object, thus maintaining the Cartesian subject-object separation that is inherent in representationalism. Heidegger wrote that this view characterized "... intentionality as an extant relation between two things extant, a psychological subject and a physical object. The nature as well as the mode of being of intentionality is completely missed (pp. 60-61)." The second misconception was the "erroneous subjectivization of intentionality. ... Intentionality is neither objective nor subjective in the usual sense, although it is certainly both (pp. 63-65). This misconception is common among psychologists who conceive intention as purpose, a mental state of goal-directedness.

Here again is the core problem: understanding the relation between the abstractions and generalizations in the structures of brain dynamics and the material involvements that are understood, and how they are understood through and beyond "likenesses": the action potentials of neurodynamicists, the phantasms of Aquinas, and the raw sense data of psychologists. The dynamical view proposes that a self-similar hierarchy of patterns, emerging from the structures of knowledge that are stored in the synaptic tissues of the brain, is continually modified by interactions with the multiple environments of the body and brain. In some deep sense this patterned activity expresses the being that Heidegger conceived as the Dasein, 
but at present with a significant limitation that constrains intentional neurodynamics to describing only first intention that animals share with children still too young to remember their lives or to distinguish themselves from any other intentional being (Dasein). Operationally the capability is defined by the mirror test: toddlers in front of a mirror look behind it to see who is there; a few months later they watch themselves touching themselves. At present the evidence for macroscopic neurodynamics comes only from animals that cannot pass the test. Second intention in which the self reflects on the process of comprehending the likenesses provided by sensory processing early in first intention is barely touched by neurodynamicists, despite major efforts to explore consciousness and awareness. This is the domain of phenomenology. Dreyfus (2006) has described remarkably close correspondences between nonlinear brain dynamics and the basic conceptions of the dynamics of intentional behaviors as conceived by Heidegger and MerleauPonty, subject to the limitation that phenomenology can only begin with consciousness of concepts that emerge far above the raw sense data and wave packets. Owing to their entry at this high-level phenomenologists cannot reach down to the level of sensation so as to distinguish between sensation and perception, as neurophysiologists distinguish them, as shown by this exchange between Merleau-Ponty (1966) and a conference organizer:

M. Parodi. Could you tell us what is your most important contribution on this question of fact. You began with very clear examples: we think we perceive things which we really only see in part, or more or less. What, according to you, is the essential element in this operation?

M. Merleau-Ponty. To perceive is to render oneself present to something through the body. All the while the thing keeps its place within the horizon of the world, and the structurization consists in putting each detail in the perceptual horizons which belong to it. But such formulas are just so many enigmas unless we relate them to the concrete developments which they summarize.

M. Parodi. I would be tempted to say that the body is much more essential for sensation than it is for perception.

M. Merleau-Ponty. Can they be distinguished?... (p. 42)",

Clearly M. Parodi did not grasp Merleau-Ponty's position, which was that sensation did not exist as a mental process, hence "the primacy of phenomenology".

\section{Conclusions}

Contemporary approaches used by researchers to understand and model both human and machine intelligence are commonly based in search for computational and representational codes. One reason is the clarity and simplicity of logical positivist concepts describing brain activity in terms of information and symbols, compared with the relative obscurity and impenetrability of the descriptors by dynamicists and phenomenologists. For nonscientists the arcane descriptions by brain dynamicists may appear just as opaque as Heidegger's and Merleau-Ponty's prose in translation appears to scientists, but scientists have the advantage of experimental grounding in brain physiology, the interpretation of which may be facilitated by translating concepts between fields. Alternative approaches to incorporate intentionality into neurobiology include those of pragmatists such as Dewey (1914): "Actions are not reactions to stimuli; they are actions into the stimuli"; Piaget (1930) in the study of child development; Köhler (1940) using field theory; Koffka (1935) using Gestalt theory; its extension by Gibson (1979) into ecological psychology; and situated cognition (Slezak, 1995). As shown by Dreyfus (2006) these and related cognitivist approaches are still shot through with strong reliance on information theory and representationalism for construction of explanatory codes. Indeed the inventor and chief architect of the programmable serial digital computer, the backbone of artificial intelligence for manipulation of symbols in coding 
systems, von Neumann (1958), realized early the limitations of the computer model:

"Thus the outward forms of our mathematics are not absolutely relevant from the point of view of evaluating what the mathematical or logical language truly used by the central nervous system is. ... It is characterized by less logical and arithmetical depth than what we are normally used to. ... We require exquisite numerical precision over many logical steps to achieve what brains accomplish in very few short steps." (pp. 81-82)

Those few short steps can now be seen through the lens of nonlinear field neurodynamics.

Brain imaging also shows great promise as a source of new experimental data on global brain dynamics, but currently it is in a phase of empirical casuistry that in many ways resembles 19 th century phrenology, owing to lack of adequate brain theory. Psychiatrists likewise rely heavily on empirical taxonomy following the failure of Freudian theory. Numerous proposals for theory have come from neurophilosophers on one hand and from mathematicians and physical scientists on the other, but with inadequate experimental support and with derivations often too strongly Cartesian to meet the challenge. Therefore, the new techniques for acquiring macroscopic data and interpreting them on the light of updated field theory and neuropercolation theory can provide the solid conceptual structure that is necessary to solve the core problem of philosophy. There is more. Thomist-Heideggerian philosophy will likely lead to constructing a totally new class of machine, the intentional robot, which is based in neurodynamics instead of digital logic (Kozma and Freeman, 2003; Kozma et al., 2003; Dreyfus, 2006). This possibility is as relevant to philosophers as it is to engineers. If an intelligent machine can comprehend and remember only the sensory consequences of its own intended actions, then it must be equipped with appropriate sensors, effectors, sources of reward, and the autonomy to explore its environment with learning by trial and error under reinforcement. Demonstration of a solution to the core problem of cognitive science and philosophy by such modeling of first intention must precede an approach to second intention, for which there is no realistic possibility at present.

From detailed measurements of the electric fields of the brain it is possible to infer that the essential operation in the sensory cortices is to replace (transpose) stimulus input with constructs by the brain of conceptions that stem from anticipation based in memory. These constructs emerge by cooperative neurodynamics operating over a continuum of scales in time and space that can be divided into levels corresponding to the techniques of observation and measurement of brain activity and behavior. The constructs are states of knowledge that support predictions by multisensory projections from the present into the future of desired rewards through patterns of sensory input from the body and the environment. The anticipations exist as macroscopic patterns of neural activity that order ("enslave") the mesoscopic populations of neurons comprising the sensory and motor areas. In the sensory cortical areas the local attractor landscapes embody the specific predictions. The motor cortical areas embed the tactical trajectories of neural activity that control the movements of the body and with proprioception shape the actions in the context of the changing environment. The changes in sensory inflow resulting from movements are transmitted to sensory cortical areas, where they encounter the attractor landscapes formulated through preafference as internal model-building. The sensory and motor mesoscopic activity patterns that exist in the forms and trajectories of the material substrate of neural activity are the abstract concepts that govern the engagement of the Dasein with the world by anticipating, acting, sensing, generalizing, and assimilating, encompassing first intention in animals and in preconscious states of humans.

In neurodynamics the process can be studied at the multiple levels of its material substrate in brain, body, and environment and the forms pertaining thereto. In physics the process can best be described by models that combine the agent of action with that part of the environment that is engaged, creating a mirror image or 'double' in order to balance the energy flows in the unified 
system (Vitiello, 2001). In philosophy the concepts referred to as phenomena constitute the mind, which directly enters into the world on its own terms, achieving closure and "maximum grip" without intermediation by representations of raw sense data (Dreyfus, 2006). What is still inacces-

sible to analysis with respect to neurodynamics is an explanation of second intention, the awareness of experiencing of the world. There is no physiological test for consciousness even at the elemental level of that which is obtunded by anesthesia or sleep. There is only the phenomenological test of asking a subject, "What do you remember?" and comparing the answer with objective records. In the lack of such a test the only acceptable conclusion is that we do not now understand the process of self-awareness. The aim of this essay is to describe a pathway in brain dynamics toward understanding by experimental observation and measurement of the macroscopic fields of the brains of normal subjects, which will require devising and applying new and advanced EEG technology supplemented in parallel with related techniques of noninvasive brain imaging.

\section{References}

Aquinas, St. Thomas. (1272) The Summa Theologica. Translated by Fathers of the English Dominican Province. Revised by Daniel J. Sullivan. Published by William Benton as Volume 19 in the Great Books Series. Encyclopedia Britannica, Inc., Chicago, IL, 1952.

Barabásí, A.-L. (2002) Linked. The New Science of Networks. Perseus, Cambridge, MA.

Barlow, H.B. (1972) Single units and sensation: a neuron doctrine for perceptual psychology? Perception, 1: 371-394.

Braitenberg, V. and Schüz, A. (1998) Cortex: Statistics and Geometry of Neuronal Connectivity (2nd ed.). Springer-Verlag, Berlin.

Brentano, F.C. (1889/1969) The Origin of Our Knowledge of Right and Wrong. Chisolm R.M. and Schneewind E.H. (trans.). Humanities Press, New York.

Bressler, S.L. and Kelso, J.A.S. (2001) Cortical coordination dynamics and cognition. Trends Cogn. Sci., 5: 2-36.

Buck, L. and Axel, R. (2004) A novel multigene family may encode odorant receptors: a molecular basis for odor recognition. Cell, S116(2): 175-187.

Burr, C. (2002) The Emperor of Scent: A Story of Perfume, Obsession, and the Last Mystery of the Senses. Random House, New York.
Buzsaki, G. (2002) Theta oscillations in the hippocampus. Neuron, 33(3): 325-340.

Chalmers, D.J. (1996) The Conscious Mind: In Search of a Fundamental Theory. Oxford University Press, New York.

Dewey, J. (1914) Psychological doctrine in philosophical teaching. J. Philos., 11: 505-512.

Dreyfus, H. (2006) Why Heideggerian AI failed and how fixing it would require making it more Heideggerian. Inquiry, to be published.

Ferster, C.B. and Skinner, B.F. (1957) Schedules of Reinforcement. Prentice-Hall, Englewood Cliffs, NJ.

Freeman, W.J. (1975/2004) Mass Action in the Nervous System. Academic Press, New York http://sulcus.berkeley.edu/ MANSWWW/MANSWWW.html.

Freeman, W.J. (1995) Societies of Brains. A Study in the Neuroscience of Love and Hate. Lawrence Erlbaum, Mahwah, NJ.

Freeman, W.J. (2001) The olfactory system: odor detection and classification. In: Frontiers in Biology, Vol. 3. Intelligent Systems. Part II. Brain Components as Elements of Intelligent Function. Academic Press, New York, pp. 509-526.

Freeman, W.J. (2004a) Origin, structure, and role of background EEG activity. Part 1. Analytic amplitude. Clin. Neurophysiol., 115: 2077-2088.

Freeman, W.J. (2004b) Origin, structure, and role of background EEG activity. Part 2. Analytic phase. Clin. Neurophysiol., 115: 2089-2107.

Freeman, W.J. (2005a) Origin, structure, and role of background EEG activity. Part 3. Neural frame classification. Clin. Neurophysiol., 116(5): 1118-1129.

Freeman, W.J. (2005b) A field-theoretic approach to understanding scale-free neocortical dynamics. Biol. Cybern., 92(6): 350-359.

Freeman, W.J. (2005c) NDN, volume transmission, and selforganization in brain dynamics. J. Integr. Neurosci., 4(4): 407-421.

Freeman, W.J. (2006a) Origin, structure, and role of background EEG activity. Part 4. Neural frame simulation. Clin. Neurophysiol., 117(3): 572-589.

Freeman, W.J. (2006b) Definitions of state variables and state space for brain-computer interface. Part 1. Multiple hierarchical levels of brain function. Cogn. Neurodyn., 1(1): 18713080 (print); 1871-4099 (online) http://dx.doi.org/10.1007/ s11571-006-9001-x

Freeman, W.J. (2007a) Hilbert transform for brain waves. Scholarpedia. p. 7514. http://www.scholarpedia.org/article/ Hilbert_transform_for_brain_waves

Freeman, W.J. (2007b) Intentionality. Scholarpedia. p. 8616. http://www.scholarpedia.org/article/Intentionality

Freeman, W.J. (2007c) Scale-free neocortical dynamics. Scholarpedia. p. 8780. http://www.scholarpedia.org/article/ScaleFree_Neocortical_Dynamics

Freeman, W.J. and Burke, B.C. (2003) A neurobiological theory of meaning in perception. Part 4. Multicortical patterns of amplitude modulation in gamma EEG. Int. J. Bifurc. Chaos, 13: 2857-2866. 
Freeman, W.J., Burke, B.C. and Holmes, M.D. (2003a) Aperiodic phase re-setting in scalp EEG of beta-gamma oscillations by state transitions at alpha-theta rates. Hum. Brain Mapp., 19(4): 248-272.

Freeman, W.J., Burke, B.C., Holmes, M.D. and Vanhatalo, S. (2003b) Spatial spectra of scalp EEG and EMG from awake humans. Clin. Neurophysiol., 114: 1055-1060 http://repositories.cdlib.org/postprints/989.

Freeman, W.J. and Rogers, L.J. (2003) A neurobiological theory of meaning in perception. Part 5. Multicortical patterns of phase modulation in gamma EEG. Int. J. Bifurc. Chaos, 13: 2867-2887.

Freeman, W.J. and Vitiello, G. (2006) Nonlinear brain dynamics as macroscopic manifestation of underlying many-body field dynamics. Phys. Life Rev., 3: 93-118.

Gibson, J.J. (1979) The Ecological Approach to Visual Perception. Houghton Mifflin, Boston, MA

Haken, H. (1983) Synergetics: An Introduction. Springer, Berlin.

Heidegger, M. (1975/1988) The Basic Problems of Phenomenology (rev. ed.). Hofstadter A. (trans.). Indiana University Press, Bloomington, IN.

Houk, J.C. (2005) Agents of the mind. Biol. Cybern., 92: $427-437$.

Houk, J.C. and Wise, S.P. (1995) Distributed modular architectures linking basal ganglia, cerebellum, and cerebral cortex: their role in planning and controlling action. Cereb. Cortex, 5: 95-110.

Kay, L.M. and Freeman, W.J. (1998) Bidirectional processing in the olfactory-limbic axis during olfactory behavior. Behav. Neurosci., 112: 541-553.

O'Keefe, J.M. and Nadel, L. (1978) The Hippocampus as a Cognitive Map. Oxford University Press, New York.

Koffka, K. (1935) Principles of Gestalt Psychology. Harcourt Brace, New York.

Köhler, W. (1940) Dynamics in Psychology. Grove Press, New York.

Kozma, R. and Freeman, W.J. (2003) Basic principles of the KIV model and its application to the navigation problem. J. Integr. Neurosci., 2: 125-145.

Kozma, R., Freeman, W.J. and Erdí, P. (2003) The KIV model - nonlinear spatio-temporal dynamics of the primordial vertebrate forebrain. Neurocomputing, 52: 819-826 http:// repositories.cdlib.org/postprints/1049.

Kozma, R., Puljic, M., Balister, P., Bollabás, B. and Freeman, W.J. (2005) Phase transitions in the neuropercolation model of neural populations with mixed local and non-local interactions. Biol. Cybern., 92: 367-379 http://repositories.cdlib.org/postprints/999.

Lancet, D. and Ben-Arie, N. (1993) Olfactory receptors. Curr. Biol., 3: 6768-6774.

Lettvin, J.Y. and Gesteland, R.C. (1965) Speculations on smell. Cold Spring Harbor Symp. Quant. Biol., 30: 217-225.

Maclean, P.D. (1969) The Triune Brain. Plenum, New York.

Merleau-Ponty, M. (1942/1963) The Structure of Behavior. Fischer A.L. (trans.). Beacon, Boston, MA.

Merleau-Ponty, M. (1966) The Primacy of Perception. In: Edie J.M. (Ed.). Northwestern University Press, Evanston, IL.

von Neumann, J. (1958) The Computer and the Brain. Yale University Press, New Haven, CT.

Piaget, J. (1930) The child's conception of physical causality. Harcourt, Brace, New York.

Prigogine, I. (1980) From Being to Becoming: Time and Complexity in the Physical Sciences. WH Freeman, San Francisco, CA.

Quiroga, Q.R., Reddy, L., Kreiman, G., Koch, C. and Fried, I. (2005) Invariant visual representation by single-neurons in the human brain. Nature, 435: 1102-1107.

Searle, J.R. (1983) Intentionality. Cambridge University Press, Cambridge, UK.

Sherrington, C.S. (1906) The Integrative Action of the Nervous System. Yale University Press, New Haven, CT.

Skarda, C.A. and Freeman, W.J. (1987) How brains make chaos in order to make sense of the world. Behav. Brain Sci., 10: $161-195$.

Slezak, P. (1995) The 'philosophical' case against visual imagery. In: Slezak P., Caelli T. and Clark R. (Eds.), Perspectives on Cognitive Science: Theories, Experiments and Foundations. Ablex Publ., Greenwich, CT, pp. 237-271.

Sperry, R.W. (1950) Neural basis of the spontaneous optokinetic response. J. Comp. Physiol., 43: 482-489.

Tsuda, I. (2001) Toward an interpretation of dynamics neural activity in terms of chaotic dynamical systems. Behav. Brain Sci., 24: 793-847.

Turin, L. (2006) The Secret of Scent. HarperCollins, New York. Vitiello, G. (2001) My Double Unveiled. John Benjamins, Amsterdam.

Von Holst, E. and Mittelstädt, H. (1950) Das Reafferenz Prinzip. Wechselwirkung zwischen Zentralnervensystem und Peripherie. Naturewissenschaften, 37: 464-476. 


\begin{tabular}{|c|c|c|}
\hline 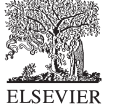 & $\begin{array}{l}\text { Book: PBR-V165 } \\
\text { Chapter: } 65028\end{array}$ & $\begin{array}{l}\text { Please eail or fax your responses and any corrections to: } \\
\text { Eail: } \\
\text { Fax: }\end{array}$ \\
\hline
\end{tabular}

Dear Author,

During the preparation of your manuscript for typesetting, some questions may have arisen. These are listed below. Please check your typeset proof carefully and mark any corrections in the margin of the proof or compile them as a separate list*.

\section{Disk use}

Sometimes we are unable to process the electronic file of your article and/or artwork. If this is the case, we have proceeded by:

$\square$ Scanning (parts of) your article $\square$ Rekeying (parts of) your article

$\square$ Scanning the artwork

\section{Bibliography}

If discrepancies were noted between the literature list and the text references, the following may apply:

The references listed below were noted in the text but appear to be missing from your literature list. Please complete the list or remove the references from the text.

$\square$ Uncited references: This section comprises references that occur in the reference list but not in the body of the text. Please position each reference in the text or delete it. Any reference not dealt with will be retained inthis section

Queries and/or remarks

\begin{tabular}{|l|l|l|}
\hline $\begin{array}{l}\text { Location in } \\
\text { Article }\end{array}$ & Query/ remark & Response \\
\hline AU:1 & $\begin{array}{l}\text { The references Freeman (1999, } \\
\text { 2000) are not listed in the } \\
\text { reference list. Please provide } \\
\text { complete details of the reference. }\end{array}$ & $\begin{array}{l}\text { Since there are two publications } \\
\text { of Freeman et al. (2003), as per } \\
\text { style, we cross-refer it as '2003a' } \\
\text { and '2003b' for tagging purposes. } \\
\text { Please go through each citation } \\
\text { and indicate whether the } \\
\text { reference is 2003a or 2003b. }\end{array}$ \\
\hline AU:3 & $\begin{array}{l}\text { Please provide the ref. Freeman } \\
\text { (in press) in the reference list with } \\
\text { complete details. }\end{array}$ & \\
\hline AU:4 & $\begin{array}{l}\text { Please provide complete details } \\
\text { of reference Dreyfus (2006). }\end{array}$ & \\
\hline
\end{tabular}

